\title{
Prognostic capabilities of coronary computed tomographic angiography before non-cardiac surgery: prospective cohort study
}

\begin{abstract}
Tej Sheth, ${ }^{1}$ Matthew Chan, ${ }^{2}$ Craig Butler, ${ }^{3}$ Benjamin Chow, ${ }^{4}$ Vikas Tandon, ${ }^{5}$ Peter Nagele, ${ }^{6}$ Ayesha Mitha, ${ }^{7}$ Marko Mrkobrada, ${ }^{8}$ Wojciech Szczeklik, ${ }^{9}$ Yang Faridah, ${ }^{10}$ Bruce Biccard, ${ }^{11}$ Lori K Stewart, ${ }^{12}$ Diane Heels-Ansdell, ${ }^{13}$ P J Devereaux, ${ }^{14}$ on behalf of the Coronary Computed Tomographic Angiography and Vascular Events in Noncardiac Surgery Patients Cohort Evaluation (Coronary CTA VISION) Study Investigators
\end{abstract}

\section{ABSTRACT \\ OBJECTIVES}

To determine if coronary computed tomographic angiography enhances prediction of perioperative risk in patients before non-cardiac surgery and to assess the preoperative coronary anatomy in patients who experience a myocardial infarction after non-cardiac surgery.

DESIGN

Prospective cohort study.

SETTING

12 centers in eight countries.

PARTICIPANTS

955 patients with, or at risk of, atherosclerotic disease who underwent non-cardiac surgery.

INTERVENTIONS

Coronary computed tomographic angiography was performed preoperatively; clinicians were blinded to the results unless left main disease was suspected. Results were classified as normal, non-obstructive ( $<50 \%$ stenosis), obstructive (one or two vessels with

\section{WHAT IS ALREADY KNOWN ON THIS TOPIC}

Clinical risk prediction models provide suboptimal prediction of risk of perioperative cardiac complications

The incremental risk prediction of imaging tests is uncertain because of limited availability of high quality evidence

Prognosis is poor for myocardial infarction after non-cardiac surgery, but there is limited knowledge regarding the degree of coronary artery stenosis in patients who have a perioperative myocardial infarction

\section{WHAT THIS STUDY ADDS}

Compared with the revised cardiac risk index alone, findings on preoperative coronary computed tomographic angiography will appropriately improve risk estimation among patients who will experience perioperative cardiovascular death or a myocardial infarction but will inappropriately result in overestimation of risk among patients who will not experience these outcomes within 30 days of non-cardiac surgery

With 30 day risk categories of $<5 \%, 5-15 \%$, and $>15 \%$ for the primary outcome, the net absolute effect in a sample of 1000 patients is that coronary computed tomographic angiography will result in an inappropriate estimate of risk in 81 patients compared with risk estimation based on the revised cardiac risk index alone.

Perioperative myocardial infarction occurs in patients with and without preoperative obstructive coronary artery disease on preoperative coronary computed tomographic angiography $\geq 50 \%$ stenosis), or extensive obstructive ( $\geq 50 \%$ stenosis in two vessels including the proximal left anterior descending artery, three vessels, or left main).

\section{MAIN OUTCOME MEASURE}

Composite of cardiovascular death and non-fatal myocardial infarction within 30 days after surgery (primary outcome). This was the dependent variable in Cox regression. The independent variables were scores on the revised cardiac risk index and findings on coronary computed tomographic angiography.

\section{RESULTS}

The primary outcome occurred in 74 patients (8\%). The model that included both scores on the revised cardiac risk index and findings on coronary computed tomographic angiography showed that coronary computed tomographic angiography provided independent prognostic information ( $\mathrm{P}=0.014$; $C$ index $=0.66)$. The adjusted hazard ratios were $1.51(95 \%$ confidence interval 0.45 to 5.10 ) for non-obstructive disease; 2.05 ( 0.62 to 6.74 ) for obstructive disease; and 3.76 (1.12 to 12.62) for extensive obstructive disease. For the model with coronary computed tomographic angiography compared with the model based on the categories of $<5 \%, 5-15 \%$, and $>15 \%$ for the primary outcome, the results of risk reclassification indicate that in a sample of 1000 patients that coronary computed tomographic angiography would have resulted appropriately in 17 net patients receiving a higher risk estimation among the 77 patients who would have experienced the primary outcome $(P<0.001)$. Coronary computed tomographic angiography, however, would have resulted inappropriately in 98 net patients receiving a higher risk estimation, among the 923 patients who would not have experienced the primary outcome $(\mathrm{P}<0.001)$. Among patients who had a coronary anatomy showed extensive obstructive disease in $31 \%$ (22/71), obstructive disease in $41 \%$ (29/71), non-obstructive disease in $24 \%$ (17/71), and normal findings in $4 \%(3 / 71)$.

\section{CONCLUSIONS}

Though findings on coronary computed tomographic angiography can improve estimation of risk for patients who will experience perioperative cardiovascular death or myocardial infarction, findings are more than five times as likely to lead to an inappropriate overestimation of risk among patients revised cardiac risk index alone, with 30 day risk perioperative myocardial infarction, preoperative 
who will not experience these outcomes. Perioperative myocardial infarction occurs across the spectrum of coronary artery disease, suggesting that there could be several pathophysiologic mechanisms.

\section{Introduction}

Although major cardiac complications after noncardiac surgery are common, ${ }^{1}$ our capacity to predict these events in individual patients is limited. ${ }^{2}$ The ability to identify an increased risk of perioperative ischemic events could influence the type of operation performed or inform the decision whether to proceed with intervention or manage conservatively. Alternatively, patients with an anticipated low risk are likely to be operated on safely without delay. ${ }^{3}$ Clinical practice guidelines recommend assessment of preoperative risk, beginning with clinical risk indices. ${ }^{3}$ Clinical risk indices, however, underestimate the risk of major perioperative complications, particularly in patients with limited mobility before surgery. ${ }^{5}$ It might, therefore, be worth using a supplemental method to enhance risk prediction. Though myocardial infarction is the most common major perioperative cardiac complication, little is known about its pathophysiology. ${ }^{2}$

Coronary computed tomographic angiography is a non-invasive method for the detection of coronary artery disease. In contrast with stress nuclear and stress echocardiography imaging, which detect areas of ischemic myocardium, coronary computed tomographic angiography identifies the presence and distribution of coronary plaque and stenoses. In patients with stable angina, the extent of coronary artery disease shown on coronary computed tomographic angiography can predict the occurrence of major cardiac events. ${ }^{6-8}$ Its value in enhancing risk prediction among patients undergoing non-cardiac surgery, however, is unknown.

We conducted this study (the coronary computed tomographic angiography vascular events in noncardiac surgery patients cohort evaluation (Coronary CTA VISION)) to determine if preoperative coronary computed tomographic angiography has additional predictive value, beyond clinical variables, for cardiovascular death or non-fatal myocardial infarction within 30 days after surgery and the preoperative coronary anatomy associated with perioperative myocardial infarction.

\section{Methods}

\section{Study design and eligibility criteria}

This was a prospective observational study. We have published details of the study objectives, design, and methods elsewhere. ${ }^{9}$

Patients were eligible if they fulfilled the following criteria: age $\geq 45$; undergoing elective vascular, orthopedic, thoracic, or abdominal surgery in hospital; had sufficient time to undergo coronary computed tomographic angiography before surgery; and had a history of, or risk factors for, atherosclerotic disease or a history of congestive heart failure.

Patients fulfilling any of the following criteria were excluded: planned invasive coronary angiography for preoperative investigation before surgery; history of coronary artery stent implantation; creatinine clearance $<35 \mathrm{~mL} / \mathrm{min}$; known contrast reaction; current pregnancy; persistent atrial fibrillation or frequent premature beats; heart rate $\geq 70$ beats/min (at centers with single source scanners) or $\geq 90$ beats/min (at centers with dual source scanners), despite drugs to control heart rate just before scheduled coronary computed tomographic angiography; weight $>300 \mathrm{lb}$ (136 kg); more than four non-evaluable segments on coronary computed tomographic angiography (non-diagnostic scan); did not undergo non-cardiac surgery within six months after coronary computed tomographic angiography; surgery that did not require at least an overnight stay in hospital; or results of coronary computed tomographic angiography were unblinded because of suspected left main stenosis and patient underwent preoperative coronary revascularization.

\section{Coronary computed tomographic angiography}

The protocol we used for coronary computed tomographic angiography imaging is reported in appendix 1. A panel of expert evaluators (that is, a cardiologist or radiologist with level 3 training in interpretation of coronary computed tomographic angiography) read each angiogram using a 17 segment model of the coronary arteries without knowledge of the clinical data. ${ }^{10}$ Each of scans was scored as normal-no evidence of coronary atherosclerosis; non-obstructive coronary artery disease-evidence of at least one coronary artery plaque with a $<50 \%$ stenosis; obstructive coronary artery disease-at least one coronary artery plaque with $\mathrm{a} \geq 50 \%$ stenosis; or extensive obstructive disease $-\geq 50 \%$ stenosis in two coronary arteries including the proximal left anterior descending artery, $\geq 50 \%$ stenosis in three coronary arteries, or $\geq 50 \%$ stenosis in the left main coronary artery. ${ }^{9}$

Patients who had previously undergone coronary artery bypass grafting surgery were assessed for the number of unprotected coronary territories (bypass graft with $\geq 50 \%$ stenosis and native coronary artery with $\geq 50 \%)$. ${ }^{11}$ Patients with no or one unprotected coronary territory were classified as having obstructive coronary artery disease, and patients with two or three unprotected coronary territories were classified as having extensive obstructive disease.

Patients with $a \geq 50 \%$ stenosis in the left main artery had the results of their coronary computed tomographic angiography reported immediately to their attending physicians. Potentially important incidental non-cardiac findings were disclosed immediately after the scan was interpreted. All other patients had their results withheld from the attending clinical care team until 30 days after surgery.

\section{Study procedures}

Study personnel obtained data on patients' characteristics. All patients had scheduled troponin measurements at six to 12 hours after surgery and on the first, second, and third days after surgery. An electrocardiogram was obtained immediately after an increased troponin measurement was detected. 
Study personnel followed patients throughout their hospital stay and reviewed their medical records ensuring study orders were followed and noting any outcomes (such as mortality). We contacted patients by phone 30 days after surgery. If patients or their next of kin indicated that they had experienced an outcome (that is, myocardial infarction or mortality) or had been admitted to hospital, study personnel obtained the appropriate documentation from the attending clinicians.

\section{Outcomes measures and definitions}

Our primary outcome was a composite of cardiovascular death and non-fatal myocardial infarction within 30 days after surgery. For the diagnosis of myocardial infarction we used the criteria of the universal definition of myocardial infarction ${ }^{12}$ that required a typical rise of troponin concentration associated with one of the following: ischemic signs or symptoms, ischemic changes on electrocardiography, or new imaging abnormalities suggestive of myocardial infarction. A panel of clinicians who were blinded to the results of coronary computed tomographic angiography adjudicated the outcomes of cardiovascular death and myocardial infarction. We used the adjudicated results for all statistical analyses.

\section{Patient involvement}

We did not involve patients or lay people in the design of the study, selection of outcome measures, or recruitment plans and do not plan to disseminate the results to the study participants.

\section{Statistical analyses}

A priori, we determined that we needed 1000 patients to ensure a stable model if our primary event rate was $6 \% .{ }^{9}$ After we had 30 day outcomes for 950 patients, we determined that our event rate was $>7.5 \%$ and that we had enough patients to assess whether coronary computed tomographic angiography provided independent prognostic information beyond clinical variables. We therefore stopped recruitment without knowledge of the relation between the findings and the primary outcome and subsequently undertook the analyses. The data monitoring committee reviewed the data when about $40 \%$ of the data and separately when $65 \%$ of the data on 30 day outcomes were available.

The operations committee prespecified the data analysis plan. Patients who did not complete 30 day follow-up were censored on the last day that their vital status was known. We determined the percentage of patients who had a primary outcome event within 30 days after surgery.

We undertook Cox proportional hazards modeling in which the dependent variable was cardiovascular death and non-fatal myocardial infarction. In the first model, the independent variable was the score on the revised cardiac index..$^{13}$ This risk score is well validated, widely used, and recommended for clinical risk stratification in recent guidelines. ${ }^{34}$ The model includes six variables: high risk surgery, history of coronary artery disease, history of heart failure, history of cerebrovascular disease, preoperative treatment with insulin, and preoperative creatinine $>170 \mathrm{mmol} / \mathrm{L}$. The presence of each variable adds one point, and patients obtain scores from 0-6, with higher scores indicating greater risk. ${ }^{13}$

In the second model, the independent variable was findings on coronary computed tomographic angiography (that is, non-obstructive, obstructive, and extensive obstructive with a reference category of normal coronary arteries). The final model included both the clinical risk scores on the revised cardiac risk index and findings of coronary computed tomographic angiography as independent variables.

For all independent predictors of the primary outcome, we determined the adjusted hazard ratio and the associated 95\% confidence intervals. A two sided $P$ value was designated a priori as significant at an $\alpha$ of 0.05. Discrimination was assessed through evaluation of the $\mathrm{C}$ index. Likelihood ratios were determined for each category of findings on coronary computed tomographic angiography.

We performed post hoc sensitivity analyses to determine if the results were influenced by whether or not a patient had a history of prior vascular disease, had prior coronary artery disease, or had undergone vascular or orthopedic surgery. For these analyses, we used an interaction term in our models for each of these factors. We also undertook another post hoc subgroup analysis restricted to patients without a history of a prior coronary artery bypass grafting.

We calculated reclassification of risk among patients who experienced the primary outcome and separately among patients who did not experience the primary outcome to determine if findings on coronary computed tomographic angiography improved risk classification beyond that achieved with the revised cardiac risk index clinical model. ${ }^{14}$ In this analysis, we classified the 30 day primary outcome as low risk $<5 \%$, intermediate risk $5-15 \%$, and high risk $>15 \%$. We also performed a sensitivity analysis for the net improvement in reclassification that included four risk categories ( $<5 \%, 5-10 \%$, $\geq 10-15 \%$, and $\geq 15 \%$ ) and a post hoc evaluation of the risk categories recommended in the European Society of Cardiology guidelines $(<1 \%, 1-5 \%$, and $>5 \%)$. $^{3}$

We performed post hoc analyses to evaluate the prognostic capabilities of coronary computed tomographic angiography using a $\geq 70 \%$ stenosis threshold to define obstructive coronary artery and extensive obstructive. We also performed post hoc analyses to evaluate the prognostic capabilities of coronary computed tomographic angiography in patients with one or two of the following: history of coronary artery disease, age $>70$, and diabetes requiring treatment. ${ }^{15}$ All analyses were performed with SAS version 9.2 (SAS Institute, Cary, NC).

\section{Results}

Patients were recruited from 12 centers from July 2008 to October 2013. Of the 1093 patients who underwent preoperative coronary computed tomographic 


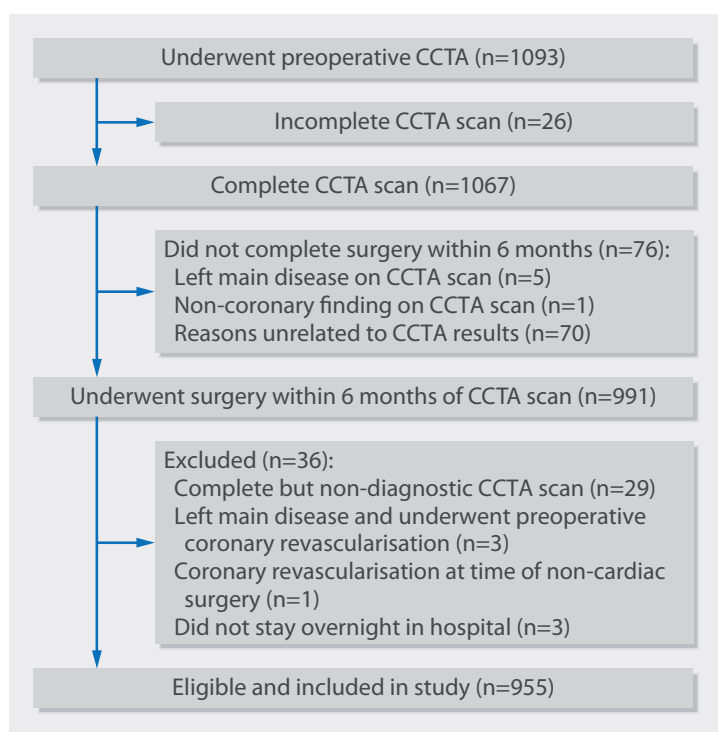

Fig 1 | Flow of patients throughout study of prognostic capabilities of coronary computed tomographic angiography carried out before non-cardiac surgery (CCTA=coronary computed tomographic angiography)

angiography, 1067 underwent a scan. We identified 14 patients with suspected left main disease and unblinded their angiography results. Five of these patients had their non-cardiac surgery cancelled. A further 71 patients did not undergo surgery within six months for other reasons and were also excluded (fig 1). Of the remaining 991 patients, three were excluded because they had left main disease and underwent preoperative coronary revascularization and 33 were excluded because they had a non-diagnostic scan, outpatient surgery, or had concomitant coronary revascularization with non-cardiac surgery.

The 955 remaining eligible patients were enrolled and included in the study. We included six of the 14 patients with suspected left main disease because these patients had non-cardiac surgery without preoperative coronary revascularization. Among the included patients, $99.5 \%$ completed the 30 day follow-up.

Table 1 shows characteristics of the patients and types of surgery. The mean age was 69.7 and $61 \%$ (584/955) were men. A history of known vascular disease was present in 61\% (579/955) of patient, and 39\% (376/955) of patients had three or more vascular risk factors without a history of known vascular disease. The most common score on the revised cardiac risk index was 1 (43\%, 407/955), and $24 \%$ (228/955) of patients had a score $\geq 2$. The most common types of surgery were orthopedic (56\%, 538/955) and vascular (30\%, 289/955).

Coronary computed tomographic angiography was performed with prospective triggering in 33\% (319/955) of patients and with retrospective gating with $\mathrm{x}$ ray dose modulation in 53\% (501/955). The mean contrast volume was $100.2 \mathrm{~mL}$, and the mean radiation dose, measured as the dose length product, was $797.6 \mathrm{mGy}-\mathrm{cm}$ (or $11.2 \mathrm{mSV}$ ).

Results of angiography showed that 81/955 patients (8\%) had normal coronary arteries, 371/955 (39\%) had non-obstructive coronary artery disease, 357/955 (37\%) had obstructive coronary artery disease, and 146/955 (15\%) had extensive obstructive disease. The primary outcome (that is, cardiovascular death or myocardial infarction) occurred in $74 / 955$ patients (8\%), of whom eight (1\%) experienced cardiovascular death and 71 (7\%) experienced a myocardial infarction. Fig 2 shows the Kaplan Meier curves for the primary outcome by findings on coronary computed tomographic angiography. The primary outcome occurred in 3/81 patients (4\%) with normal findings, 19/371 (5\%) with non-obstructive coronary artery disease, 29/357 (8\%) with obstructive coronary artery disease, and 23/146 (16\%) with extensive obstructive disease. None of the six patients with suspected left main stenosis, who underwent non-cardiac surgery without preoperative revascularization, experienced the primary outcome. Kaplan Meier curves for cardiovascular death and myocardial infarction separately are reported figs A and B in appendix 2.

Table 2 shows the models to predict 30 day cardiovascular death and myocardial infarction. In the model with scores on the revised cardiac risk index as the only independent variables, the C index was 0.62 (95\% confidence interval 0.56 to 0.68 ). A score $\geq 3$ compared with a score of 0 had a hazard ratio of 5.25 (95\% confidence interval 2.41 to 11.43 ). In the model with findings on coronary computed tomographic angiography as the only independent variables, the $\mathrm{C}$ index was 0.62 (0.56 to 0.68). Extensive obstructive coronary artery disease compared with normal findings resulted in a hazard ratio of 4.50 (1.35 to 15.00). In the model that included both coronary computed tomographic angiography and the revised cardiac risk index, the $\mathrm{C}$ index was 0.66 (0.60 to 0.73). In this model, that included scores on the revised cardiac risk index, extensive obstructive coronary artery disease had an adjusted hazard ratio of 3.76 (1.12 to 12.62). The likelihood ratios for findings on coronary computed tomographic angiography are reported in table A in appendix 3. Extensive obstructive disease had a likelihood ratio of 2.23 (1.53 to 3.24).

The post hoc sensitivity analyses to determine if the ability of coronary computed tomographic angiography to provide independent prognostic information varied by subgroups showed no significant effect of a history of vascular disease (interaction $\mathrm{P}=0.984$ ), a history of coronary artery disease (interaction $\mathrm{P}=0.327$ ), or type of surgery (interaction $\mathrm{P}=0.325$ ). For patients without prior coronary artery bypass grafting, the findings on coronary computed tomographic angiography in our model that included scores on the revised cardiac risk index are shown in table B in appendix 3. The hazard ratio point estimates for the findings on coronary computed tomographic angiography in patients who did not undergo prior coronary artery bypass grafting were similar to the results for the entire study population.

For the model that included the findings on coronary computed tomographic angiography compared with the model that included only the scores on the revised cardiac risk index, the reclassification of risk was $22 \%$ 


\begin{tabular}{|c|c|c|c|}
\hline Demographics & All patients $(n=955)$ & $\begin{array}{l}\text { Did not experience } \\
\text { cardiovascular } \\
\text { death or myocardial } \\
\text { infarction }(n=881)\end{array}$ & $\begin{array}{l}\text { Experienced } \\
\text { cardiovascular } \\
\text { death or myocardial } \\
\text { infarction }(n=74)\end{array}$ \\
\hline Mean (SD) age (years) & $69.7(8.5)$ & $69.5(8.6)$ & $72.2(7.9)$ \\
\hline Men & $584(61)$ & $536(61)$ & $48(65)$ \\
\hline Mean (SD) BMI & $30.0(6.2)$ & $30.1(6.3)$ & $28.6(5.0)$ \\
\hline \multicolumn{4}{|l|}{ Known vascular disease: } \\
\hline History of CAD & $305(32)$ & $267(30)$ & $38(51)$ \\
\hline History of PVD & $332(35)$ & $295(33)$ & $37(50)$ \\
\hline History of stroke & $88(9)$ & $78(9)$ & $10(14)$ \\
\hline History of CHF & $35(4)$ & $30(3)$ & $5(7)$ \\
\hline Any of the above & $579(61)$ & $518(59)$ & $61(82)$ \\
\hline \multicolumn{4}{|l|}{ Vascular risk factors: } \\
\hline History of diabetes & $362(38)$ & $328(37)$ & $34(46)$ \\
\hline Age $\geq 70$ & $512(54)$ & $464(53)$ & $48(65)$ \\
\hline History of smoking within 2 years of surgery & $257(27)$ & $239(27)$ & $18(24)$ \\
\hline History of treatment for hypercholesterolemia & $766(80)$ & $711(81)$ & $55(74)$ \\
\hline History of TIA & $91(10)$ & $83(9)$ & $8(11)$ \\
\hline History of hypertension & $838(88)$ & $770(87)$ & $68(92)$ \\
\hline $\begin{array}{l}\text { Patients qualified based on risk factors alone (that is, } \geq 3 \text { of } \\
6 \text { risk factors) }\end{array}$ & $376(39)$ & $363(41)$ & $13(18)$ \\
\hline Recent high risk CAD & $8(1)$ & $6(1)$ & $2(3)$ \\
\hline Myocardial infarction & 1 & 1 & 0 \\
\hline CCS II Angina & 7 & 5 & 2 \\
\hline \multicolumn{4}{|l|}{ Patients with baseline: } \\
\hline Stress nuclear imaging & $90(9)$ & $78(9)$ & $12(16)$ \\
\hline Stress echocardiography & $7(1)$ & $7(1)$ & 0 \\
\hline Patient requires assistance with activities of daily living & $40(4)$ & $36(4)$ & $5(5)$ \\
\hline \multicolumn{4}{|l|}{ Patients with baseline factors before operation: } \\
\hline Statins $<24 \mathrm{~h}$ & $457(48)$ & $424(48)$ & $33(45)$ \\
\hline Statins $>24 \mathrm{~h}$ to 7 days & $705(74)$ & $650(74)$ & $55(74)$ \\
\hline$\beta$ blocker $<24 \mathrm{~h}$ & $294(31)$ & $263(30)$ & $31(42)$ \\
\hline$\beta$ blocker $>24 h$ to 7 days & $369(39)$ & $326(37)$ & $43(58)$ \\
\hline \multicolumn{4}{|l|}{ Revised cardiac risk index score*: } \\
\hline 0 & $320(34)$ & $305(35)$ & $15(20)$ \\
\hline 1 & 407 (43) & $378(43)$ & $29(39)$ \\
\hline 2 & $178(19)$ & 159 (18) & $19(26)$ \\
\hline 3 & $43(5)$ & $33(4)$ & $10(14)$ \\
\hline 4 & $6(1)$ & $6(1)$ & 0 \\
\hline 5 & $1(<1)$ & 0 & $1(1)$ \\
\hline 6 & 0 & 0 & 0 \\
\hline \multicolumn{4}{|l|}{ Type of surgery: } \\
\hline Vascular & $289(30)$ & $259(29)$ & $30(41)$ \\
\hline Intra-abdominal & $72(8)$ & $67(8)$ & $5(7)$ \\
\hline Orthopedic & $538(56)$ & $502(57)$ & $36(49)$ \\
\hline Major spine & $13(1)$ & $13(1)$ & 0 \\
\hline Other & $53(6)$ & $49(6)$ & $4(5)$ \\
\hline
\end{tabular}

(16/74; $95 \%$ confidence interval $10 \%$ to $33 \%$; $\mathrm{P}<0.001$ ) for those who experienced the primary outcome and $-11 \%$ ( $-94 / 881 ;-14 \%$ to $-7 \%$; $\mathrm{P}<0.001)$ for those who did not experience the primary outcome (table 3$)$. When we compared the model with coronary computed tomographic angiography with the model based on the revised cardiac risk index alone, using 30 day risk categories of $<5 \%, 5-15 \%$, and $>15 \%$, the results of risk reclassification indicate that in a sample of 1000 patients that coronary computed tomographic angiography would have resulted appropriately in 17 net patients receiving a higher risk estimation, among the 77 patients who would have experienced the primary outcome $(\mathrm{P}<0.001)$; however, coronary computed tomographic angiography would have resulted inappropriately in 98 net patients receiving a higher risk estimation, among the 923 patients who would not have experienced the primary outcome $(\mathrm{P}<0.001)$. The overall net effect in a sample of 1000 patients would be that coronary computed tomographic angiography would result in an inappropriate 


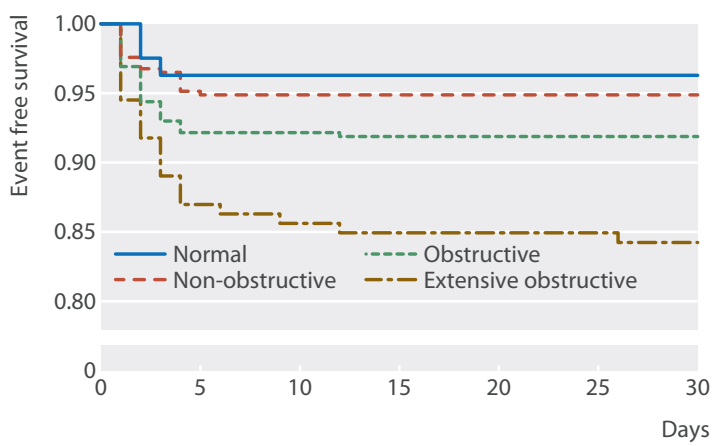

Fig 2 | Findings on coronary computed tomographic angiography and survival free from cardiovascular death or myocardial infarction

estimate of risk in 81 patients compared with risk estimation based on the revised cardiac risk index alone.

The sensitivity analysis with four categories of risk suggested that the net effect in a sample of 1000 patients would be that coronary computed tomographic angiography would result in a net inappropriate estimate of risk in 22 patients compared with risk estimation based on the revised cardiac risk index alone (table $\mathrm{C}$ in appendix 3). Table $\mathrm{D}$ in appendix 3 reports the post hoc risk reclassification analyses for the risk categories proposed in the European Society of Cardiology guidelines $(<1 \%, 1-5 \%$, and $>5 \%))^{3}$ The findings were similar to those of our a priori primary risk categories.
Table E in appendix 3 reports the post hoc sensitivity analysis evaluating the impact of using $\geq 70 \%$ stenosis to define obstructive and extensive obstructive coronary artery disease. The associated post hoc sensitivity analysis for the risk reclassification indicates that in a sample of 1000 patients, coronary computed tomographic angiography would result in a net overall inappropriate estimate of risk in 34 patients compared with risk estimation based on the revised cardiac risk index alone (table $\mathrm{F}$ in appendix 3). Table $\mathrm{G}$ in appendix 3 reports the post hoc risk reclassification analyses that included only patients with one or two of the following risk factors: history of coronary artery disease, age $>70$, diabetes requiring treatment. The risk reclassification in those with an event was $20.4 \%(\mathrm{P}<0.001)$ and in those without an event was $-12.4 \%(\mathrm{P}<0.001)$. These results were therefore similar to the overall study.

Among patients experiencing a myocardial infarction, preoperative coronary computed tomographic angiography imaging showed extensive obstructive disease in 31\% (22/71), obstructive disease in 41\% (29/71), non-obstructive disease in 24\% (17/71), and normal results in 4\% (3/71) (see fig $\mathrm{C}$ in appendix 2).

\section{Discussion}

\section{Principal findings}

In this international prospective blinded cohort study of 955 patients, multivariable analysis showed that compared with the revised cardiac risk index alone, findings on preoperative coronary computed tomographic

Table 2 | Models to predict 30 day cardiovascular death and myocardial infarction in study of prognostic capabilities of coronary computed tomographic angiography carried out before non-cardiac surgery

\begin{tabular}{|c|c|c|c|c|}
\hline Model type & $\begin{array}{l}\text { No of } \\
\text { patients }\end{array}$ & $\begin{array}{l}\text { No with } \\
\text { event (\%) }\end{array}$ & $\begin{array}{l}\text { Hazard ratio } \\
(95 \% \mathrm{Cl})\end{array}$ & Pvalue \\
\hline \multicolumn{5}{|c|}{ Revised cardiac risk index scores as only independent variables* } \\
\hline \multicolumn{5}{|c|}{ RCRI scores (overall $P<0.001 \dagger$ ): } \\
\hline 0 & 320 & $15(4.7)$ & 1.00 & - \\
\hline 1 & 407 & $29(7.1)$ & $1.53(0.82$ to 2.86$)$ & 0.178 \\
\hline 2 & 178 & $19(10.7)$ & $2.37(1.20$ to 4.66$)$ & 0.013 \\
\hline$\geq 3$ & 50 & $11(22.0)$ & $5.25(2.41$ to 11.43$)$ & $<0.001$ \\
\hline \multicolumn{5}{|c|}{ CCTA findings as only independent variables* } \\
\hline \multicolumn{5}{|c|}{ CCTA findings (overall $P<0.001 \ddagger$ ): } \\
\hline Normal & 81 & $3(3.7)$ & 1.00 & - \\
\hline Non-obstructive & 371 & $19(5.1)$ & $1.40(0.41$ to 4.72$)$ & 0.590 \\
\hline Obstructive & 357 & $29(8.1)$ & $2.26(0.69$ to 7.41$)$ & 0.180 \\
\hline Extensive obstructive & 146 & $23(15.8)$ & $4.50(1.35$ to 15.00$)$ & 0.014 \\
\hline \multicolumn{5}{|c|}{ Combined CCTA findings and revised cardiac risk index scores§ } \\
\hline \multicolumn{5}{|c|}{ RCRI scores (overall $P=0.005 t$ ): } \\
\hline 0 & 320 & $15(4.7)$ & 1.00 & - \\
\hline 1 & 407 & $29(7.1)$ & 1.39 (0.74 to 2.61$)$ & 0.300 \\
\hline 2 & 178 & $19(10.7)$ & $1.88(0.94$ to 3.79$)$ & 0.076 \\
\hline$\geq 3$ & 50 & $11(22.0)$ & $4.02(1.80$ to 8.98$)$ & $<0.001$ \\
\hline \multicolumn{5}{|c|}{ CCTA findings (overall $P=0.014 \ddagger$ ): } \\
\hline Normal & 81 & $3(3.7)$ & 1.00 & - \\
\hline Non-obstructive & 371 & $19(5.1)$ & $1.51(0.45$ to 5.10$)$ & 0.509 \\
\hline Obstructive & 357 & $29(8.1)$ & $2.05(0.62$ to 6.74$)$ & 0.238 \\
\hline Extensive obstructive & 146 & $23(15.8)$ & $3.76(1.12$ to 12.62$)$ & 0.032 \\
\hline \multicolumn{5}{|c|}{$\begin{array}{l}\text { CCTA=coronary computed tomographic angiography; } R C R I=\text { revised cardiac risk index. } \\
{ }^{*} \mathrm{C}=0.62,95 \% \text { confidence interval } 0.56 \text { to } 0.68 \text {. } \\
\text { †Overall P value for four category } R C R I \text {. } \\
\text { ‡Overall P value for four category CCTA. } \\
\S C=0.66,95 \% \text { confidence interval } 0.60 \text { to } 0.73 \text {. }\end{array}$} \\
\hline
\end{tabular}




\begin{tabular}{|c|c|c|c|c|c|c|}
\hline \multirow[b]{3}{*}{ RCRI scores only } & \multicolumn{6}{|c|}{ Model that included CCTA findings } \\
\hline & \multicolumn{3}{|c|}{ Patients who had an event } & \multicolumn{3}{|c|}{ Patients who did not have an event } \\
\hline & $<5 \%$ & $5 \%-15 \%$ & $>15 \%$ & $<5 \%$ & $5 \%-15 \%$ & $>15 \%$ \\
\hline$<5 \%$ & 5 & 10 & 0 & 191 & 114 & 0 \\
\hline $5 \%-15 \%$ & 0 & 41 & 7 & 47 & 453 & 37 \\
\hline$>15 \%$ & 0 & 1 & 10 & 0 & 10 & 29 \\
\hline \multicolumn{7}{|c|}{$\begin{array}{l}{ }^{*} \text { CCTA risk reclassification for those who experienced primary outcome was: }(17-1) / 74=22 \%, 95 \% \text { confidence } \\
\text { interval } 10 \% \text { to } 33 \% \text {; } P<0.001 \text {. Risk reclassification for those who did not experience primary outcome was: } \\
(57-151) / 881=-11 \%,-14 \% \text { to }-7 \% ; P<0.001 \text {. }\end{array}$} \\
\hline
\end{tabular}

angiography can appropriately improve risk estimation among patients who will experience perioperative cardiovascular death or have a myocardial infarction. Coronary computed tomographic angiography, however, is more than five times as likely to inappropriately overestimate risk among patients who will not experience cardiovascular death or a myocardial infarction within 30 days of non-cardiac surgery. Among patients who experienced myocardial infarction after surgery, we observed considerable variation in the extent of preoperative coronary artery disease.

\section{Strengths and limitations of our study}

Our study has several methodological strengths. We enrolled a large number of patients with a wide spectrum of clinical risk at multiple centers. Our results are therefore likely to be widely generalisable. With the exception of suspected left main stenosis, all results from coronary computed tomographic angiography were blinded until 30 days after surgery. Primary outcome events were adjudicated centrally by clinicians who were blinded to the angiographic findings. Clinical variables were prospectively ascertained and adjusted for in the multivariable analyses.

There were also, however, several limitations. We excluded patients who were likely to have technically suboptimal coronary computed tomographic angiography examinations (for instance, patients with prior intracoronary stents, atrial fibrillation, or raised heart rates). Only a relatively small number of recruited patients had normal findings on coronary computed tomographic angiography. Consequently, estimation of the event rate in this group was imprecise. Although our study is one of the largest evaluations of perioperative imaging, it is possible with a larger study and more events that some of the findings on coronary computed tomographic angiography beyond extensive obstructive disease could have been independently associated with the primary outcome. The risk categories of $<5 \%, 5-15 \%$, and $>15 \%$ for the primary outcome that we used to assess risk reclassification are arbitrary. These values were selected a priori because we believe they are values that physicians and patients consider important when regarding decisions around the appropriateness of surgery or requirements for enhanced perioperative monitoring. These thresholds have been used in studies evaluating the prognostic capabilities of B-type natriuretic peptides to predict cardiovascular death and non-fatal myocardial infarction in patients undergoing non-cardiac surgery. ${ }^{16}{ }^{17}$ Moreover, sensitivity and post hoc analyses with other thresholds produced qualitatively similar findings.

\section{Comparison with other modalities}

In an effort to improve risk prediction in patients undergoing non-cardiac surgery, researchers have evaluated the prognostic capabilities of non-invasive cardiac stress tests. ${ }^{18}$ We hypothesized that coronary computed tomographic angiography could be advantageous for preoperative imaging because it does not require exercise or pharmacologic stress and has high sensitivity for detection of coronary stenosis, ${ }^{19-21}$ including left main and multivessel coronary disease..$^{22}$ In our study, the model with coronary computed tomographic angiographic findings alone was able to predict the risk of cardiovascular death and myocardial infarction. The $\mathrm{C}$ index was 0.62 , with a progressive increase in the estimated hazard ratios with increasing burdens of coronary artery disease. The hazard ratio, however, was significant only for extensive obstructive disease.

Although coronary computed tomographic angiography is an anatomic imaging modality, there are important similarities between our results and those obtained in prior perioperative functional imaging studies with stress echocardiography and nuclear imaging. ${ }^{2324}$ First, highly abnormal results that substantially increase estimated risk were seen in a minority of patients (that is, we observed extensive obstructive disease in only $15 \%$ of patients). In a meta-analysis of preoperative imaging studies, moderate to severe abnormalities were seen in $16 \%$ of stress echocardiogram tests and $15 \%$ of thallium imaging tests. ${ }^{23}$ Second, milder functional abnormalities, while much more common, did not significantly increase risk. We observed a non-significant hazard ratio of 2.26 for obstructive coronary artery disease, a finding that was seen in $37 \%$ of patients. In a metaanalysis of preoperative nuclear imaging studies, $<40 \%$ ischemic myocardium was seen in $34 \%$ of patients, with likelihood ratios ranging from 1.3 to 2.9.24 Third, in our study $28 \%$ of events occurred in patients without obstructive coronary artery disease. Similarly, it has been observed that preoperative nuclear abnormalities are absent in about $30 \%$ of patients who have a perioperative myocardial infarction. ${ }^{24}$

The results of our study differ from some prior perioperative imaging studies in that our estimated hazard ratios and $\mathrm{C}$ indices were lower. For example, in the only prior study of coronary computed tomographic angiography, 11 cardiovascular events occurred among the 37 patients who had multivessel disease (adjusted odds ratio $7.31,95 \%$ confidence interval 2.25 to 23.69 ). ${ }^{25}$ This study was, however, unblinded, retrospective, and did not include routine evaluation of postoperative cardiac biomarkers to detect myocardial infarction. Although the investigators used a broad primary outcome, they had only 19 events, putting the multivariable model at risk of an overfitted result. ${ }^{26}$ In contrast, we conducted a prospective blinded study that included 
systematic monitoring of troponin measurements after surgery, and we had a more focused primary outcome (that is, cardiovascular death and myocardial infarction), and 71 of the 74 patients who experienced a primary outcome had a myocardial infarction.

Prior studies of preoperative functional imaging have shown that the presence of more extensive ischemia predicts a higher risk of perioperative events. ${ }^{23} 24$ Most of these studies were retrospective and did not have blinded evaluation of outcomes or routine screening for myocardial infarction. The confidence intervals on risk estimates associated with high risk findings were wide and overlapped between different modalities, showing that much larger comparative studies are required to determine if true differences exist between imaging techniques.

In contrast with the non-invasive imaging studies, several studies have shown that preoperative measurement B-type natriuretic peptides in blood is a strong independent predictor of cardiovascular death and non-fatal myocardial infarction in patients undergoing non-cardiac surgery. ${ }^{1617}$ Moreover, this blood test improves risk estimation both in patients who do and do not experience cardiovascular death or myocardial infarction.

\section{Prediction beyond clinical variables}

Imaging tests are expensive and usually require an additional visit to the clinic or hospital. Coronary computed tomographic angiography and nuclear imaging also expose patients to contrast reaction and/or radiation hazards. It is therefore important to establish that imaging tests can improve risk evaluation beyond clinical variables. Most studies of preoperative imaging have not assessed whether non-invasive tests provide independent prognostic information, rather they have simply assessed the association between the imaging findings and the outcome without adjustment for the clinical information. The few studies that have assessed the independent prognostic information from imaging tests provide unreliable estimates because the analyses had too few events for the number of variables assessed. ${ }^{27-31}$

In our study, we had 74 events and over 10 events per variable assessed in our model that combined scores on the revised cardiac risk index and findings of coronary computed tomographic angiography imaging. We found that findings on coronary computed tomographic angiography imaging independently affected risk estimation. Although these angiographic findings improved risk estimation among patients who experienced the primary event, they more commonly resulted in inappropriate overestimation of risk in patients who did not experience the primary outcome. This was also true for the results based on the post hoc analysis that evaluated the impact of using $\geq 70 \%$ stenosis to define obstructive and extensive obstructive coronary artery disease.

Overestimation of risk can have negative consequences. It could lead to patients deciding against undergoing surgery because they view the risk as too high, when they would have accepted their actual risk. Clinicians might refer patients perceived to be at increased risk to invasive angiography and revascularization, which would delay surgery and is of uncertain value in the perioperative period. ${ }^{32}$ There are limited resources for monitoring the highest risk patients after surgery, and if patients are inappropriately sent to a monitored bed because of an erroneous risk estimation this could preclude access for a patient who is actually genuinely at high risk.

A potential benefit of coronary computed tomographic angiography might be the ability to detect left main stenosis, which would likely prompt coronary revascularization before non-cardiac surgery. ${ }^{33}$ In our study, we identified 14 (1\%) patients with suspected left main stenosis among 1067 who completed the coronary computed tomographic angiography scan, a detection rate that is possibly too low to justify routine such screening. Moreover, among the six patients with suspected left main stenosis who underwent non-cardiac surgery without preoperative revascularization, none experienced the primary outcome.

There is value in identifying clinical contexts in which routine imaging screening does not provide an incremental benefit in risk prediction or outcomes. Our findings suggest that the resources that would be spent on preoperative coronary computed tomographic angiography could be better utilized in other aspects of patient care. Similarly, a recent randomized controlled trial that evaluated coronary computed tomographic angiography screening in asymptomatic patients with diabetes showed no impact on cardiac events. ${ }^{34}$

\section{Coronary anatomy associated with perioperative myocardial infarction}

The pathophysiology of perioperative myocardial infarction remains controversial. ${ }^{2}$ Evaluation of coronary angiograms from patients who have already experienced a perioperative myocardial infarction suggests that both thrombosis (type 1 myocardial infarction) and supply-demand mismatch (type 2 myocardial infarction) can play a role. ${ }^{3536}$ These studies are limited by selection bias because patients with perioperative myocardial infarction often do not undergo coronary angiography.

Our study provides complimentary angiographic data on a large sample of patients before surgery, showing that $72 \%$ of patients who had a perioperative myocardial infarction had either obstructive or extensive obstructive coronary artery disease visible on preoperative coronary computed tomographic angiography. Patients with this pattern of coronary artery disease are at risk of type 1 and type 2 myocardial infarction. In contrast, no preoperative coronary stenosis $\geq 50 \%$ was seen in $28 \%$ of patients who experienced a perioperative myocardial infarction. In the absence of severe fixed coronary artery disease, mechanisms other than type 2 myocardial infarction are likely implicated. Further investigations into the pathophysiology of perioperative myocardial infarction and treatment interventions are needed. 


\section{Conclusions}

Compared with the revised cardiac risk index alone, findings on preoperative coronary computed tomographic angiographic will appropriately improve risk estimation among patients who will experience perioperative cardiovascular death or myocardial infarction but will inappropriately result in overestimation of risk among patients who will not experience such outcomes. With 30 day risk categories of $<5 \%, 5-15 \%$, and $>15 \%$ for the primary outcome, the overall net effect in a sample of 1000 patients is that coronary computed tomographic angiography will result in an inappropriate estimate of risk in 81 patients compared with risk estimation based on the revised cardiac risk index alone. Perioperative myocardial infarction occurs across the spectrum of coronary artery disease, suggesting the existence of multiple pathophysiologic mechanisms.

\section{AUTHOR AFFILIATIONS}

'Population Health Research Institute, David Braley Cardiac, Vascular, and Stroke Research Institute, Hamilton, ON L8L 2X2, Canada

2Department of Anesthesia and Intensive Care, Chinese University of Hong Kong, Hong Kong, SAR, China

${ }^{3}$ Department of Medicine, Division of Cardiology, University of Alberta, 2C2 Walter Mackenzie Centre, Edmonton, AB T6G 2B7, Canada

${ }^{4}$ Departments of Medicine (Cardiology) and Radiology, University of Ottawa Heart Institute, Ottawa, ON K1Y 4W7, Canada

${ }^{5}$ Division of Cardiology, Department of Medicine, St. Joseph's Healthcare, McMaster University, Hamilton, ON L8N 4A6, Canada

${ }^{6}$ Department of Anesthesiology, Washington University School of Medicine, St Louis, Washington, MO 63110, USA

${ }^{7}$ Inkosi Albert Luthuli Central Hospital-Department of Radiology, Cato Manor, Durban, 4091, South Africa

${ }^{8}$ Division of General Internal Medicine,University of Western Ontario, London, ON N6A 5A5, Canada

${ }^{9}$ Department of Medicine, Jagiellonian University Medical College, 31-027 Krakow, Poland

${ }^{10}$ Department of Biomedical Imaging, University Malaya Research Imaging Centre, Faculty of Medicine, University Malaya, Kuala Lumpur 50603, Malaysia

${ }^{11}$ University of Kwazulu-Natal, Glenwood, Durban, 4041, South Africa

${ }^{12}$ Diagnostic Imaging, Hamilton Health Sciences, Jurvanski Hospital, Hamilton, ON L8V 1C3, Canada

${ }^{13}$ Department of Clinical Epidemiology and Biostatistics, McMaster University, Hamilton, ON L8S 3Z5, Canada

${ }^{14}$ Departments of Clinical Epidemiology and Biostatistics and Medicine, McMaster University, Hamilton, ON L8S 3Z5, Canada

We thank the participants for enrolling in the study.

This study was coordinated by the Clinical Advances Through Research and Information Translation (CLARITY) project office and the Population Health Research Institute (PHRI), at the Hamilton Health Sciences, McMaster University, Hamilton, ON, Canada.

\section{CTA VISION Investigators}

Hamilton, Canada: L Stewart, V Tandon, M Ferri: Ottawa, Canada: BJW Chow, P Jetty; Edmonton, Canada: C Butler, R Coulden, M Graham, M Jacka; Hong Kong, China: M Chan, GYS Choi, KT Wong; Durban, South Africa: A Mitha, T Kisten, B Biccard, R Rodseth; St. Louis, US: P Nagele, P Woodard; Toronto, Canada: AM Crean; Kuala Lampur, Malaysia: C Wang, Y F Abdul Aziz, R Sandhu; Sydney, Australia: C K Chow; New Delhi, India: G Karthikeyan, G Gulati; London, Canada: M Markobrada, A Islam, A Goela; Krakow, Poland: W Szczeklik, T Miszalski-Jamka, J Gorka. Data monitoring committee

V Montori (chair), N Valettas, K Thorlund.
Contributors: TS, PJD, MC, CB, and BC were responsible for study design and concept. TS, PJD, MC, CB, BC, and DH-A analyzed and interpreted data. DH-A carried out the statistical analysis. TS, PJD, MM, and PN obtained funding. All authors acquired the data and critically revised the manuscript for important intellectual content. TS and PJD are guarantors.

Funding: This study was funded by the Canadian Institutes of Health Research, the Hamilton Health Sciences New Investigator Fund, the McMaster University Division of Cardiology, the University of Western Ontario Department of Radiology, the University of Western Ontario Division of General Internal Medicine, and the Washington University Institute of Clinical and Translational Sciences. The study funders had no role in design and conduct of the study; collection, management, analysis, and interpretation of the data; and preparation, review, or approval of the manuscript.

Competing interests: All authors have completed the Unified Competing Interest form at www.icmje.org/coi_disclosure.pdf (available on request from the corresponding author) and declare that TS has received research grants from GE Healthcare and St Jude Medical, BJC receives research support from GE Healthcare and educational support from TeraRecon, and PJD is part of a group that has a policy of not accepting honorariums or other payments from industry for their own personal financial gain. Members of this group accept honorariums or other payments from industry to support research endeavors and for reimbursement of costs to participate in meetings such as scientific or advisory committee meetings. Based on study questions PJD originated and grants he wrote, he has received grants from Abbott Diagnostics, Astra Zeneca, Bayer, Boehringer Ingelheim, Bristol-Myers Squibb, Covidien, Philips Healthcare, Stryker, and Roche Diagnostics. PJD has also participated in an advisory boarding meeting for GlaxoSmithKline and an expert panel meeting for Astra Zeneca.

Ethical approval: The study was approved by the research ethics board of each participating institution and was overseen by an independent data monitoring committee. Written informed consent was given by all patients.

Data sharing: No additional data are available.

Transparency: TS and PJD affirm that the manuscript is an honest, accurate, and transparent account of the study being reported; that no important aspects of the study have been omitted; and that any discrepancies from the study as planned have been explained. This is an Open Access article distributed in accordance with the Creative Commons Attribution Non Commercial (CC BY-NC 4.0) license, which permits others to distribute, remix, adapt, build upon this work non-commercially, and license their derivative works on different terms, provided the original work is properly cited and the use is noncommercial. See: http://creativecommons.org/licenses/by-nc/4.0/.

1 Devereaux PJ, Chan MT, Alonso-Coello P, et al. Vascular Events In Noncardiac Surgery Patients Cohort Evaluation Study I, Association between postoperative troponin levels and 30-day mortality among patients undergoing noncardiac surgery. JAMA 2012:307:2295-304

2 Devereaux PJ, Chan M, Eikelboom J. Major vascular complications in patients undergoing non-cardiac surgery: the magnitude of the problem, risk prediction, surveillance, and prevention. In: YusufS, Cairns J, Camm AJ, et al, ed. Evidence based cardiology. 3rd ed. BM] Books, 2009:47-62.

3 Kristensen SD, Knuuti J, Saraste A, et al. 2014 ESC/ESA Guidelines on non-cardiac surgery: cardiovascular assessment and management: the joint task force on non-cardiac surgery: cardiovascular assessment and management of the European Society of Cardiology (ESC) and the European Society of Anaesthesiology (ESA). Eur Heart) 2014:35:2383-431.

4 Fleisher LA, Fleischmann KE, Auerbach AD, et al. 2014 ACC/AHA guideline on perioperative cardiovascular evaluation and management of patients undergoing noncardiac surgery: a report of the American College of Cardiology/American Heart Association Task Force on practice guidelines. J Am Coll Cardiol 2014;64:e77-137.

5 Chan A, Livingstone D, Tu J. The Goldman and Detsky cardiac-risk indices: Do they work in patients undergoing hip-fracture surgery? Annals RCPSC 1999;32:337-41

6 Chow $B$, Wells GA, Chen L, et al. Prognostic value of 64-slice cardiac computed tomography severity of coronary artery disease, coronary atherosclerosis, and left ventricular ejection fraction. J Am Coll Cardiol 2010;55:1017-28.

7 Chow B], Small G, Yam Y, et al. Incremental prognostic value of cardiac computed tomography in coronary artery disease using CONFIRM: COroNary computed tomography angiography evaluation for clinical outcomes: an InteRnational Multicenter registry. Circ Cardiovasc Imaging 2011;4:463-72. 
8 Min JK, Dunning A, Lin FY, et al. Age- and sex-related differences in all-cause mortality risk based on coronary computed tomography angiography findings results from the International Multicenter CONFIRM (Coronary CT Angiography Evaluation fo Clinical Outcomes: an International Multicenter Registry) of 23,854 patients without known coronary artery disease. J Am Coll Cardiol 2011;58:849-60.

9 Sheth T, Butler C, Chow B, et al. The coronary CT angiography vision protocol: a prospective observational imaging cohort study in patients undergoing non-cardiac surgery. BMJ Open 2012;2.

10 Austen WG, Edwards JE, Frye RL, et al. A reporting system on patients evaluated for coronary artery disease. Report of the Ad Hoc Committee for Grading of Coronary Artery Disease, Council on Cardiovascular Surgery, American Heart Association. Circulation 1975;51:5-40.

11 Chow BJ, Ahmed O, Small G, et al. Prognostic value of CT angiography in coronary bypass patients. JACC Cardiovasc Imaging 2011;4:496-502.

12 Thygesen K, Alpert JS, Jaffe AS, et al. Third universal definition of myocardial infarction. J Am Coll Cardiol 2012;60:1581-98.

13 Lee TH, Marcantonio ER, Mangione CM, et al. Derivation and prospective validation of a simple index for prediction of cardiac risk of major noncardiac surgery. Circulation 1999;100:1043-9.

14 Pencina MJ, D’Agostino RB Sr, D’Agostino RB Jr, et al. Evaluating the added predictive ability of a new marker: from area under the ROC curve to reclassification and beyond. Stat Med 2008;27:157-72; discussion 207-12

15 Eagle KA, Coley CM, Newell JB, et al. Combining clinical and thallium data optimizes preoperative assessment of cardiac risk before major vascular surgery. Ann Intern Med 1989;110:859-66.

16 Rodseth RN, Biccard BM, Chu R, et al. Postoperative B-type natriuretic peptide for prediction of major cardiac events in patients undergoing noncardiac surgery: systematic review and individual patient meta-analysis. Anesthesiology 2013;119:270-83.

17 Rodseth RN, Biccard BM, Le Manach Y, et al. The prognostic value of pre-operative and post-operative B-type natriuretic peptides in patients undergoing noncardiac surgery: B-type natriuretic peptide and $\mathrm{N}$-terminal fragment of pro-B-type natriuretic peptide: a systematic review and individual patient data meta-analysis. J Am Coll Cardiol 2014:63:170-80

18 Devereaux PJ, Goldman L, Cook DJ, et al. Perioperative cardiac events in patients undergoing noncardiac surgery: a review of the magnitude of the problem, the pathophysiology of the events and methods to estimate and communicate risk. CMAl 2005;173:627-34.

19 Mollet NR, Cademartiri F, Krestin GP, et al. Improved diagnostic accuracy with 16-row multi-slice computed tomography coronary angiography. J Am Coll Cardiol 2005; 45:128-32

20 Raff GL, Gallagher MJ, O’Neill WW, et al. Diagnostic accuracy of noninvasive coronary angiography using 64-slice spiral computed tomography. I Am Coll Cardiol 2005:46:552-7.

21 Leschka S, Alkadhi H, Plass A, et al. Accuracy of MSCT coronary angiography with 64-slice technology: first experience. Eur Heart 2005;26:1482-7.

22 Sheth T, Amlani S, Ellins ML, et al. Computed tomographic coronary angiographic assessment of high-risk coronary anatomy in patients with suspected coronary artery disease and intermediate pretest probability. Am Heart / 2008;155:918-23.
23 Beattie WS, Abdelnaem E, Wijeysundera DN, et al. A meta-analytic comparison of preoperative stress echocardiography and nuclear scintigraphy imaging. Anesth Analg 2006;102:8-16.

24 Etchells E, Meade M, Tomlinson G, et al. Semiquantitative dipyridamole myocardial stress perfusion imaging for cardiac risk assessment before noncardiac vascular surgery: a metaanalysis. J Vasc Surg 2002;36:534-40

25 Ahn JH, Park JR, Min JH, et al. Risk stratification using computed tomography coronary angiography in patients undergoing intermediate-risk noncardiac surgery. I Am Coll Cardiol 2013:61:661-8.

26 Babyak MA. What you see may not be what you get: a brief, nontechnical introduction to overfitting in regression-type models. Psychosom Med 2004:66:411-21.

27 Vanzetto G, Machecourt], Blendea D, et al. Additive value of thallium single-photon emission computed tomography myocardial imaging for prediction of perioperative events in clinically selected high cardiac risk patients having abdominal aortic surgery. Am J Cardiol 1996;77:143-8.

28 Coley CM, Field TS, Abraham SA, et al. Usefulness of dipyridamolethallium scanning for preoperative evaluation of cardiac risk for nonvascular surgery. Am / Cardiol 1992;69:1280-5.

29 Brown KA, Rowen M. Extent of jeopardized viable myocardium determined by myocardial perfusion imaging best predicts perioperative cardiac events in patients undergoing noncardiac surgery. J Am Coll Cardiol 1993;21:325-30.

30 Das MK, Pellikka PA, Mahoney DW, et al. Assessment of cardiac risk before nonvascular surgery: dobutamine stress echocardiography in 530 patients. I Am Coll Cardiol 2000;35:1647-53.

31 Hashimoto J, Suzuki T, Nakahara T, et al. Preoperative risk stratification using stress myocardial perfusion scintigraphy with electrocardiographic gating. / Nucl Med 2003;44:385-90.

32 McFalls EO, Ward HB, Moritz TE, et al. Coronary-artery revascularization before elective major vascular surgery. N Engl J Med 2004;351:2795-804

33 Fleisher LA, Beckman JA, Brown KA, et al. 2009 ACCF/AHA focused update on perioperative beta blockade incorporated into the ACC/ AHA 2007 guidelines on perioperative cardiovascular evaluation and care for noncardiac surgery: a report of the American college of cardiology foundation/American heart association task force on practice guidelines. Circulation 2009;120:e169-276.

34 Muhlestein JB, Lappe DL, Lima JA, et al. Effect of screening for coronary artery disease using $\mathrm{CT}$ angiography on mortality and cardiac events in high-risk patients with diabetes: the FACTOR-64 randomized clinical trial. JAMA 2014;312:2234-43.

35 Duvall WL, Sealove B, Pungoti C, et al. Angiographic investigation of the pathophysiology of perioperative myocardial infarction. Catheter Cardiovasc Interv 2012;80:768-76.

36 Gualandro DM, Campos CA, Calderaro D, et al. Coronary plaque rupture in patients with myocardial infarction after noncardiac surgery: frequent and dangerous. Atherosclerosis 2012;222:191-5.

(c) BMJ Publishing Group Ltd 2015

Appendix 1: Protocol

Appendix 2: Supplementary figures A-C

Appendix 3: Supplementary tables A-G 